\title{
Centrosymmetric 3D Deformation Measurement using Grid Method with a Single-Camera
}

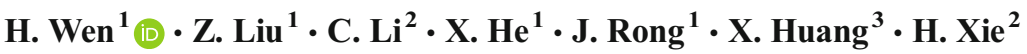

Received: 1 March 2016 / Accepted: 10 October 2016/Published online: 28 October 2016

(C) Society for Experimental Mechanics 2016

\begin{abstract}
This study proposes an effective method to measure centrosymmetric 3D static and dynamic deformations from the microscale to macroscale using grid method with a single camera. The camera was tilted at a particular angle and used to observe specimen grids in order to acquire coupling fields of both in-plane and out-of-plane displacement. This study also analyzes the decoupling methods of these two displacement types, and a systematic deduction of a theoretical equation for 3D deformation analysis was conducted based on the method. The sensitivity of morphology measurement was then evaluated and the elimination of noise and rotational errors was discussed. The efficiency and accuracy of this technique was verified through a microscale static feasibility test and a highspeed impact experiment that simulated an underwater explosion. The proposed approach uses minimal equipment, is simple and convenient, and can be used to measure centrosymmetric 3D deformation in multi-scale both statically and dynamically. In addition, this method avoids the nonsynchronization problem of a pair of high-speed cameras in high-speed 3D measurements.
\end{abstract}

\section{Z. Liu}

liuzw@bit.edu.cn

H. Xie

xiehm@mail.tsinghua.edu.cn

1 School of Aerospace Engineering, Beijing Institute of Technology, Beijing 100081, China

2 AML, Department of Engineering Mechanics, Tsinghua University, Beijing 100084, China

3 State Key Laboratory of Nonlinear Mechanics, Institute of Mechanics, Chinese Academy of Sciences, Beijing 100190, China
Keywords Centrosymmetric deformation · Grid method $\cdot 3 \mathrm{D}$ deformation $\cdot$ Micro morphology $\cdot$ Underwater explosion

\section{Introduction}

Recent technological developments have increased the demand for $3 \mathrm{D}$ deformation measurements in the microscale field or for dynamic performance characterization. To understand the mechanical properties of microstructures, the development of a method to do this in the micro/nano scale has become increasingly significant [1-4]. On the basis of previous studies, measurement methods for microstructural 3D deformation can be categorized into contact and non-contact techniques. The former generally adopts a probe to evaluate the surface topography and mechanical property of microstructures. An example of such a method is atomic force microscopy, which can measure the mechanical properties of a material and obtain its surface topography $[5,6]$. Then oncontact measurement method is suitable for micro/nano structures and has a wide measurement range and high precision. Arai used shadow moiré [7, 8] and projection moiré [9] in a Scanning Electron Microscopy (SEM) to obtain a highprecision 3D topography measurement of microstructures, but the two methods failed to show convenience in performing optimal projection angle and strain measurements. Noncontact measurement methods combined with stereovision technology have also been developed; to overcome the complexity of calibration and the influence of gray variation in 3D digital image correlation (3D DIC) proposed by Sutton [10, 11], Li [12, 13] developed 3D SEM moiré. Given that 3D DIC and 3D SEM moiré use the principle of binocular vision, both require tilting of a tested specimen to achieve different perspectives when observing the same region. Thus, before and after tilting the micro-scale specimen, a gray difference and 
location variation can occur, thereby hindering location correspondence. The aforementioned methods cannot be applied in dynamic 3D deformation measurements because of the need for a two-step tilting operation, which causes nonsynchronization before and after.

The characterization of dynamic properties of materials requires improved high-speed 3D measurement methods to simulate explosive blasts and high-speed loading in the chemical engineering and nuclear industries, among others. Since Barnard proposed computer vision technology [14] in 1982, many measurement methods for 3D deformation have been developed and applied in measuring different types of highspeed deformation and dynamic mechanical performance. These applications include random nonlinear dynamic response [15], simulation of artificial wing modal analysis of an underwing beetle [16], high-speed impact [17-19], and Hastelloy-X high-temperature vibration response [20]. However, system errors caused by the non-synchronization of the acquisition time are inevitable because of the use of a pair of high-speed cameras.

Centrosymmetry is a significant and common deformation type that occurs in almost all types of 3D deformations, such as the 3D deformation of a spherical pressure vessel or indentation [21, 22], caused by impact waves [18, 19, 23-25], and in bulge experiments [26, 27]. A multi-scale static and dynamic 3D deformation measurement technique was developed in this study on the basis of the characteristics of centrosymmetric deformation. Systematic theoretical deduction of the developed centrosymmetric 3D deformation measurement method was conducted based on a single grid image. The effectiveness of this method was verified through two experiments; static 3D deformation in microscale and an underwater explosion high-speed 3D deformation in macroscale. The technique of using a single camera has broad application prospects, especially in ultra-high-speed 3D deformation measurement for centrosymmetric, as it overcomes inconvenient operation and errors caused by using a pair of high-speed cameras, such as complex calibration processes and non-synchronization the high-speed cameras [28].

\section{Principle of Centrosymmetric 3D Deformation Measurement Based on Single-Camera and Grids}

Centrosymmetric deformation has a significant feature; the absolute values of displacement at two random points are equal if the distance between these points and the symcenter are equal. An example of this deformation can be demonstrated in a specimen with a specimen grid of horizontal and vertical lines fabricated onto a surface to be measured, as shown in Fig. 1.

Suppose that a specimen exhibits centrosymmetric 3D deformation as shown in Fig. 1, where $o$ is the symcenter. When

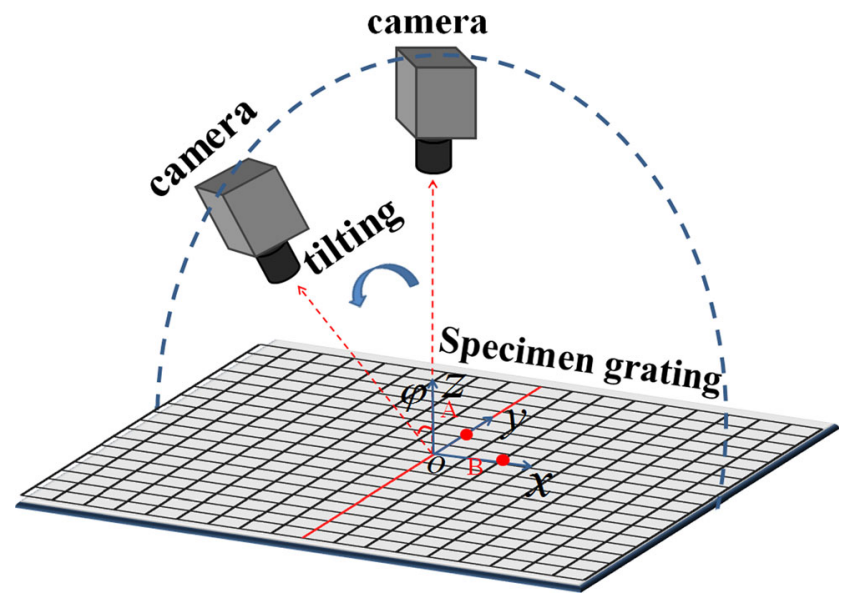

Fig. 1 An illustration showing the relative location of the specimen grid and the camera

a camera is used to observe the specimen grid and its optical axis is parallel to the $z$-axis, the absolute value of the displacement $V\left(x_{i}, y_{j}\right)$ of point $\mathrm{A}$ in the $y$-axis equals to that of the displacement $U\left(x_{i}, y_{j}\right)$ of the point $\mathrm{B}$ in the $x$-axis, if their distances from the symcenter are equal. When the camera is rotated along the $o y$ axis by an angle $\varphi$, the displacement fields $U^{\prime}\left(x_{i}, y_{j}\right)$ and $V^{\prime}\left(x_{i}, y_{j}\right)$ in the $x$ - and $y$-axes are obtained simultaneously. The displacement field $V^{\prime}\left(x_{i}, y_{j}\right)$ in the $y$-axis is not influenced by the angle of view, and the displacement field $V^{\prime}\left(x_{i}, y_{j}\right)$ is equal to $V\left(x_{i}, y_{j}\right)$. Meanwhile, the displacement field $U^{\prime}\left(x_{i}, y_{j}\right)$ in the $x$-axis is influenced by the angle of view $\varphi$ and height information $h\left(x_{i}, y_{j}\right)$; in addition, the displacement field $U^{\prime}\left(x_{i}, y_{j}\right)$ is the coupling field of the in-plane displacement (in the whole paper, in-plane refers to the specimen plane) $U\left(x_{i}, y_{j}\right)$ and height field $h\left(x_{i}, y_{j}\right)$. The height information can be obtained by analyzing the displacement fields $U^{\prime}\left(x_{i}, y_{j}\right)$ and $V\left(x_{i}, y_{j}\right)$. The specific theoretical equation for measuring the height field was deduced in the following analysis. It is notable that the effect of rotating the specimen or tilting the camera is always equivalent in the experiment, thus both the rotating specimen and tilting camera are applied in this investigation when describing the principle.

When the observation angle changes as shown in Fig. 2, the image size of the tested specimen on the imaging plane of the camera also changes. When the camera optical axis is parallel to the $z$-axis, the projected size of the specimen plane on the imaging plane is marked as $l_{1}$ and $l_{2}$, as shown in Fig. 2(a). Either before or after the centrosymmetric 3D deformation, the specimen is rotated by an angle $\varphi$ around the oy axis. The projected length in the $y$ direction is identical to the original length, namely, $l_{1}$, which remains unchanged. While, the original length $l_{2}$ in the $x$ direction transforms into the projected length $l_{2}^{\prime}$, as shown in Fig. 2(b) and (c). Thus, in Fig. 2(c), the in-plane displacement in the $y$ direction does not change before and after the rotation of the specimen, 


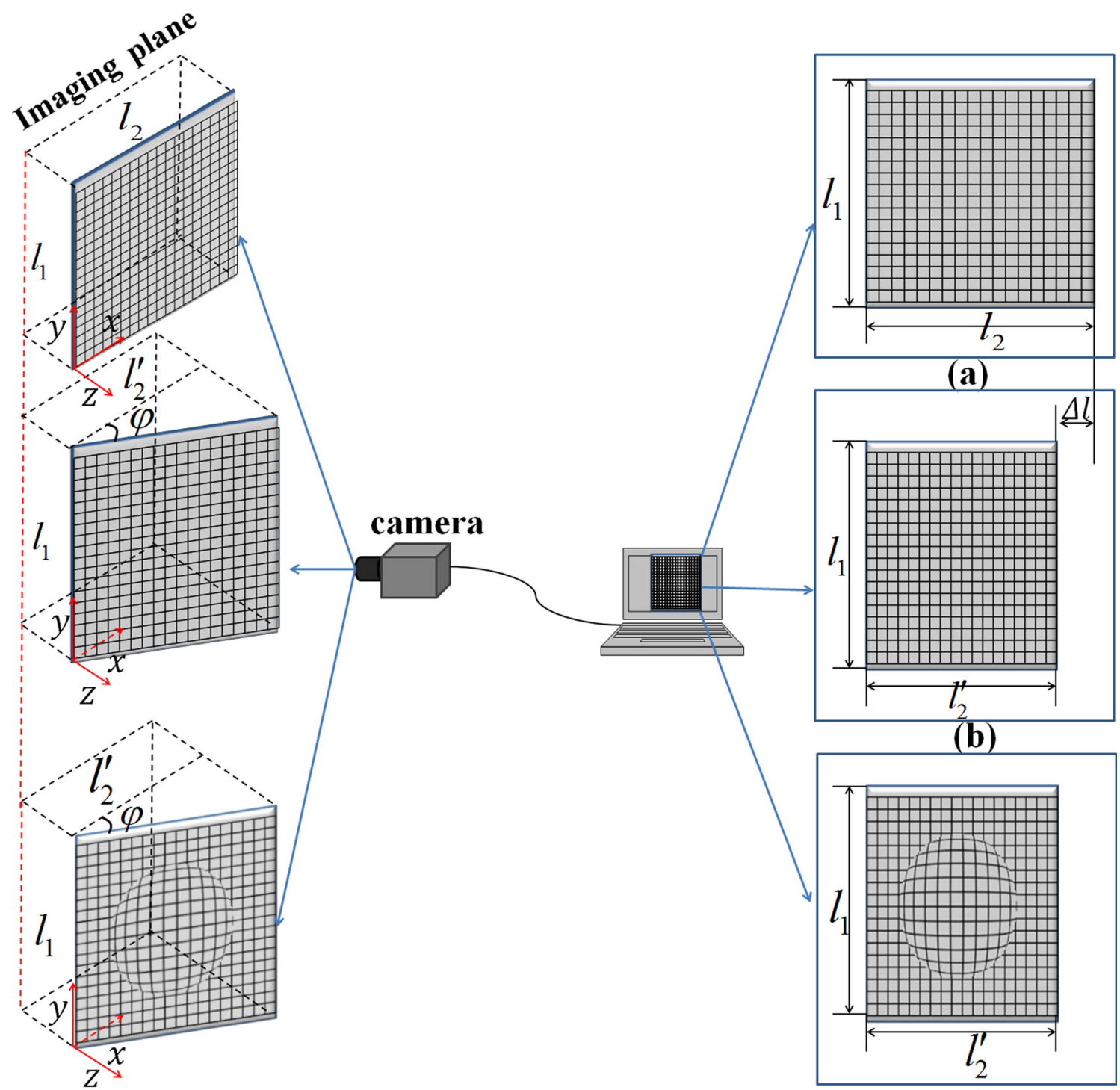

(c)

Fig. 2 An illustration of the change in picture size caused by the variation in relative location of the grid specimen grid and camera

whereas that in the $x$ direction it could change significantly depending on the angle $\varphi$.

When observing a specimen that undergoes centrosymmetric $3 \mathrm{D}$ deformation from an angle $\varphi$, it is similar to when the specimen is rotated by an angle $\varphi$ when the observing angle is unchanged, as shown in Fig. 3(a). Across section $O A B$ of the tested specimen through the symcenter can be simplified further, as shown in Fig. 3(b). After rotation, the arbitrary $\operatorname{arc} A B$ transforms into $A^{\prime} B^{\prime}$, and the following equation can be obtained through the geometric relation analysis from Fig. 3(b):

$$
\left\{\begin{array}{l}
\widehat{A B}=A^{\prime} B^{\prime} \approx S \\
l_{2}\left(x_{A B}, Y_{A B}\right)=S \cos \theta \\
l_{2}^{\prime}\left(x_{A^{\prime} B^{\prime}}, y_{A^{\prime} B^{\prime}}\right)=S \cos \beta \\
\beta=\theta
\end{array}\right.
$$

where $S$ is the chord length of $A B$ and $A^{\prime} B^{\prime} ; \theta$ is the angle between line $A B$ and the $o x$ axis; $\beta$ is the angle between line $A^{\prime}$ $B^{\prime}$ and the $o x$ axis; $l_{2}\left(x_{A B}, y_{A B}\right)$ is the projected length of $A B$; and $l_{2}^{\prime}\left(x_{A^{\prime} B^{\prime}}, y_{A^{\prime} B^{\prime}}\right)$ is the projected length of $A^{\prime} B^{\prime}$. Equation (1) can be simplified as follows:

$l_{2}^{\prime}\left(x_{A^{\prime} B^{\prime}}, y_{A^{\prime} B^{\prime}}\right)=S \cos \beta=\frac{l_{2}\left(x_{A B}, y_{A B}\right)}{\cos \theta} \cos (\varphi-\theta)$

Supposing that all deformations in this study are small deformations, the following equation can thus be derived:

$l_{2}^{\prime}\left(x_{A^{\prime} B^{\prime}}, y_{A^{\prime} B^{\prime}}\right)=\frac{l_{2}\left(x_{A B}, y_{A B}\right)}{\cos \theta} \cos (\varphi-\theta) \approx l_{2}\left(x_{A B}, y_{A B}\right) \cos \varphi(\theta<<\varphi \cdot \cos \theta \approx 1)$ 
Fig. 3 A schematic diagram showing the position relation of the centrosymmetric deformation object before and after rotation

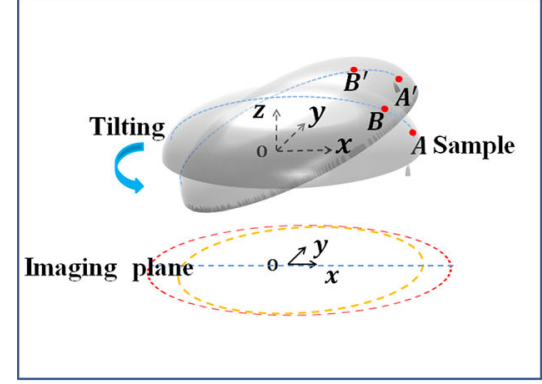

(a)

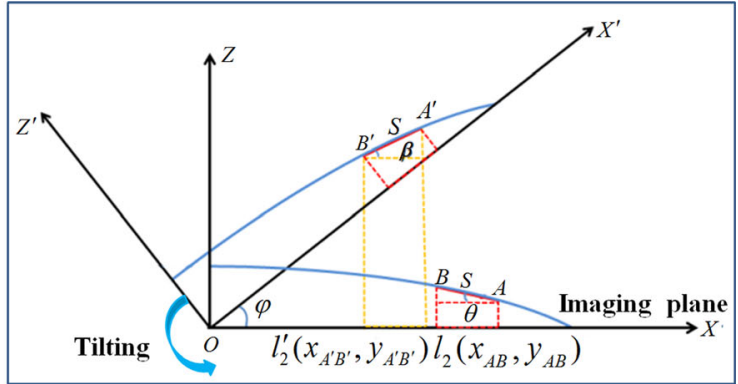

(b)
Then the following can be obtained:

$l_{2}^{\prime}=l_{2} \cos \varphi$
A schematic diagram of the $3 \mathrm{D}$ deformation measurement principle using a single-camera is shown in Fig. 4. If the tested specimen with a specimen grid undergoes centrosymmetric

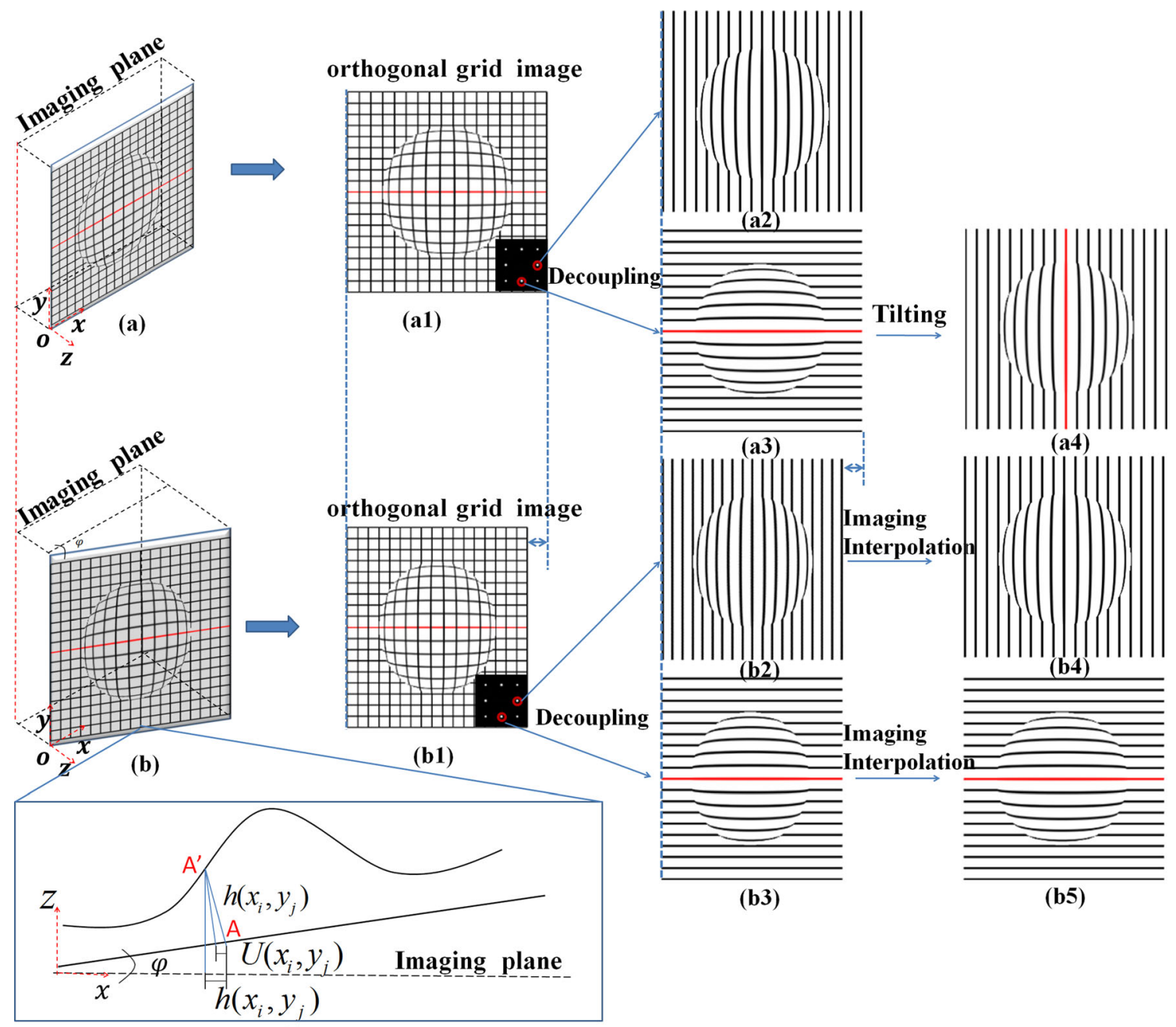

(c)

Fig. 4 A schematic diagram showing the 3D deformation measurement principle using a single camera: (a) is the specimen grid as observed from angle 0 ; (a1) is the grid after deformation; (a2) and (a3) are the decoupled unidirectional grid images in two perpendicular directions; (a4) is (a3) rotated by $90^{\circ} ;(\mathbf{b})$ is the specimen grid observed from angle $\varphi ;(\mathrm{b} 1)$ is the projected image of grid (b) on the imaging plane; (b2) and (b3) are the decoupled unidirectional grid images in two directions; (b4) and (b5) are evenly stretched images of (b2) and (b3) respectively; and (c) is the projection of a cross-section of the deformation surface 
3D deformation, then each grid image captured can be decoupled into two unidirectional grid images that are mutually vertical by using a fast Fourier transform and inverse fast Fourier transform. In Fig. 4(a) and (a1) to (a4), the optical axis of the camera is parallel to the $o z$ axis, and the displacement fields $U\left(x_{i}, y_{j}\right)$ in the $x$-axis and $V\left(x_{i}, y_{j}\right)$ in the $y$-axis can be obtained by calculating the decoupled unidirectional grid images shown in Fig. 4(a2) and (a3). In accordance with the characteristics of centrosymmetric deformation, when Fig. $4(\mathrm{a} 3)$ is rotated by $90^{\circ}$ around the symcenter and Fig. 4(a4) is obtained, the displacement field $V\left(x_{i}, y_{j}\right)$ in Fig. 4(a4) has a similar distribution as that of the displacement field $U\left(x_{i}, y_{j}\right)$ in Fig. 4(a2). The $U\left(x_{i}, y_{j}\right)$ in Fig. 4(a2) can be obtained directly by $V\left(x_{i}, y_{j}\right)$ in Fig. 4(a4). If the specimen grid is observed from angle $\varphi$ in Fig. 4(b), Fig. 4(b1) is the projected image of grid 4(b) on the imaging plane; Fig. 4(b2) and (b3) are the decoupled unidirectional grid images of Fig. 4(b1). As shown in Fig. 4(b3), the displacement field $V^{\prime}\left(x_{i}, y_{j}\right)$ can be obtained from the decoupled unidirectional grid images. Figure 4(b2) can be used to calculate the 'virtual' displacement field $U^{\prime}\left(x_{i}, y_{j}\right)$, which is the coupling field that contains information of the displacement field $U\left(x_{i}, y_{j}\right)$ and height field $h\left(x_{i}, y_{j}\right)$. Meanwhile, the displacement field $U\left(x_{i}, y_{j}\right)$ can be obtained by rotating the displacement field $V^{\prime}\left(x_{i}, y_{j}\right)$ in Fig. 4(b3) and (b5) by $90^{\circ}$. The height information $h\left(x_{i}, y_{j}\right)$ can then be deduced. Generally, the in-plane displacement field $U\left(x_{i}, y_{j}\right)$ can be directly obtained, and the height information $h\left(x_{i}, y_{j}\right)$ can be decoupled using a single specimen-grid image captured at a viewing angle of $\varphi$.

Geometric Phase Analysis (GPA) was used to calculate all the displacement fields herein. The GPA method was first introduced by Hÿtch [29, 30] and has already been applied successfully in the displacement and strain field analysis of artificial periodic lattice structures $[31,32]$. The executing processes of GPA are introduced briefly with a more detail overview available in [30]. In this paper, the reference lattice phase is $2 \pi g \cdot x$ or $2 \pi g \cdot y$. The phase is related simply to the displacement field $U^{\prime}\left(x_{i}, y_{j}\right)$ and $V^{\prime}\left(x_{i}, y_{j}\right)$ by the following equation:

$$
\left\{\begin{array}{l}
P_{g}\left(x_{i}\right)=\frac{2 \pi}{g}\left[x_{i}-U^{\prime}\left(x_{i}, y_{j}\right)\right] \\
P_{g}\left(y_{j}\right)=\frac{2 \pi}{g}\left[y_{j}-V^{\prime}\left(x_{i}, y_{j}\right)\right]
\end{array}\right.
$$

Where $g$ is the reciprocal lattice vector of the grids, and $P_{g}\left(x_{i}\right)$ and $P_{g}\left(y_{j}\right)$ are the phase fields in the $x$ and $y$ directions. In accordance with the geometric relation in Fig. 4(c) on the imaging plane, when the specimen undergoes centrosymmetric deformation and is observed at viewing angle $\varphi$, point $A$ on the specimen surface becomes point $A^{\prime}$. The following equation can then be derived through GPA for the grid deformation.

$$
\left\{\begin{array}{l}
U^{\prime}\left(x_{i}, y_{j}\right)=U\left(x_{i}, y_{j}\right) \cos \varphi+h\left(x_{i}, y_{j}\right) \sin \varphi \\
U^{\prime}\left(x_{i}, y_{j}\right)=x_{i}-\frac{g}{2 \pi} P_{g}\left(x_{i}\right) \\
V^{\prime}\left(x_{i}, y_{j}\right)=y_{j}-\frac{g}{2 \pi} P_{g}\left(y_{j}\right) \\
U\left(x_{i}, y_{j}\right)=V_{1}^{\prime}\left[f_{1}\left(x_{i}, y_{j}\right), f_{2}\left(x_{i}, y_{j}\right)\right]=\operatorname{rot} 90^{\circ}\left[V^{\prime}\left(x_{i}, y_{j}\right)\right] \\
f_{1}\left(x_{i}, y_{j}\right)=\sqrt{x_{i}^{2}+y_{j}^{2}} \cos \left(\arctan \frac{y_{j}}{x_{i}}+90\right) \\
f_{2}\left(x_{i}, y_{j}\right)=\sqrt{x_{i}^{2}+y_{j}^{2}} \sin \left(\arctan \frac{y_{j}}{x_{i}}+90\right)
\end{array}\right.
$$

On the basis of the above analysis, the displacement field $U\left(x_{i}, y_{j}\right)$ can be replaced by the displacement field $V_{1}^{\prime}\left[f_{1}\left(x_{i}, y_{j}\right), f_{2}\left(x_{i}, y_{j}\right)\right]$, which is obtained by rotating the displacement field $V^{\prime}\left(x_{i}, y_{j}\right)$ around the symcenter by $90^{\circ}$. Height information can then be obtained by further simplification:

$h\left(x_{i}, y_{j}\right)=\frac{U^{\prime}\left(x_{i}, y_{j}\right)-U\left(x_{i}, y_{j}\right) \cos \varphi}{\sin \varphi}=\frac{U^{\prime}\left(x_{i}, y_{j}\right)-V_{1}^{\prime}\left[f_{1}\left(x_{i}, y_{j}\right), f_{2}\left(x_{i}, y_{j}\right)\right] \cos \varphi}{\sin \varphi}$
$h\left(x_{i}, y_{j}\right)=\sec \varphi\left[x_{i}-\frac{g}{2 \pi} P_{g}\left(x_{i}\right)\right]-\left\{f_{2}\left(x_{i}, y_{j}\right)-\frac{g}{2 \pi} P_{g}\left[f_{2}\left(x_{i}, y_{j}\right)\right]\right\} \cot \varphi$

In this investigation, it is not necessary to directly measure the viewing angle $\varphi$. Indeed, $\varphi$ can be obtained by measuring $l_{2}$ and $l_{2}^{\prime}$ with equation (4), in which $l_{2}$ is equal to $l_{1}$. Typically, $l_{2}$ and $l_{2}^{\prime}$ are more than 100 pixels in specimen grid images, with a measuring absolute error of $\Delta l_{2}$ and $\Delta l_{2}^{\prime}$ guaranteeing less than 1 pixel. In addition, the displacement measurement sensitivity is no more than $\frac{g}{100}$ by using GPA [18], note $g$ also could bring an uncertainty and the measuring relative error of g guarantees less than $1 \%$ through repeated measurement [33]. Thus, the height measurement relative error with equation (7) is:

$$
\begin{aligned}
& \left|\frac{\Delta h}{h}\right|=\left|\frac{\Delta P_{g}\left(x_{i}\right)}{P_{g}\left(x_{i}\right)}\right|+\left|\frac{\Delta P_{g}\left(f_{2}\right)}{P_{g}\left(f_{2}\right)}\right|+2 \cdot \frac{\Delta g}{g}+\frac{\left|\Delta l_{2}\right|}{l_{2}}+\frac{\left|\Delta l_{2}^{\prime}\right|}{l_{2}^{\prime}} \\
& \quad<1 \%+1 \%+2 \%+1 \%+1 \%=6 \%
\end{aligned}
$$

While, for actual experiments, the combined standard uncertainty can be calculated using the uncertainty formulas in reference [33].

The aforementioned analysis indicates that the specimen undergoes centrosymmetric deformation; the in-plane strain 
distribution in the $x$ direction in total equals to that in the $y$ direction after rotation by $90^{\circ}$. Thus, the equation for measuring the in-plane strain field is:

$$
\begin{aligned}
& \varepsilon_{x x}\left(x_{i}, y_{j}\right)=\frac{\partial U\left(x_{i}, y_{j}\right)}{\partial x_{i}}=1-\frac{g}{2 \pi} \frac{\partial P_{g}\left(x_{i}\right)}{\partial x_{i}} \\
& \varepsilon_{y y}\left(x_{i}, y_{j}\right)=\frac{\partial V\left(x_{i}, y_{j}\right)}{\partial y_{j}}=1-\frac{g}{2 \pi} \frac{\partial P_{g}\left(y_{j}\right)}{\partial y_{j}} \\
& \varepsilon_{x x}\left(x_{i}, y_{j}\right)=\operatorname{rot} 90^{\circ}\left[\frac{\partial V^{\prime}\left(x_{i}, y_{j}\right)}{\partial y_{j}}\right]=\operatorname{rot} 90^{\circ}\left[\varepsilon_{y y}^{\prime}\left(x_{i}, y_{j}\right)\right]
\end{aligned}
$$

Not all observing angles are appropriate as the limitation of the viewing angle is related to the reciprocal lattice vector $g$, height information $h\left(x_{i}, y_{j}\right)$, and computing sensitivity of the displacement computing method. The minimum viewing angle can be determined from:

$\varphi \geq \min \left[\arcsin \left(\frac{g}{100} \cdot \frac{1}{h\left(x_{i}, y_{j}\right)}\right)\right]$

To ensure the accuracy of the developed method, the sources of measurement error and how to eliminate them are discussed in detail here. The two main types of error are noise error and rotational angle error. The noise error was related to the quality of the surface grid fabrication and the imaging system used; the quality of the surface grid was dependent on the quality of the grid pitch and the pitch error of the laser marking system here was no more than $1 \%$. Imaging distortion caused by the imaging system error was always present no matter which imaging system was used, such as camera lens distortion in a CCD chip and nonlinear higher order distortion in SEM. Using a parametric distortion model can correct lens distortion [34]. Based on the non-parametric distortion model [35], a correction method for nonlinear higher order distortion terms using DIC metrology proposed by Sutton [10] and the secondary moiré method could effectively eliminate the imaging distortion in SEM. Here, a relative simple method was used to remove the noise error: Because there was zero distortion in the center of image, in order to reduce imaging system error, the central region of the tested specimen needed to be placed near the center of the whole field of view before testing.

In this paper, the distortion deformation caused by a noise error $U_{\text {noise }}$ contains that of the grid fabricating error $U_{\text {grating }}$ and the imaging system error $U_{\text {distort }}$. Because $U_{\text {grating }}$ and
$U_{\text {distort }}$ almost do not vary during the whole experiment, the displacement field caused by $U_{\text {grating }}$ and $U_{\text {distort }}$ can be regarded as an original carrier $U_{\text {carrier }}$ before, during and after loading. The real displacement can be obtained from the deformed displacement field by directly subtracting the original carrier:

$U_{\text {real }}=U_{\text {deform }}-U_{\text {carrier }}$

The rotational error was caused by the camera rotation or direction of the specimen grid. Both have the same influence; the specimen grid in the imaging plane may not be completely parallel to the horizontal or vertical direction. In this case, the rotational error can be reduced by actually measuring the angle between the grid line and horizontal or vertical direction and adjusting the specimen or camera to make the angle error small enough to be ignored. Even if the rotational error cannot be ignored, the displacement distribution in the $x$ and $y$ directions can be corrected automatically using the GPA method [36]. The noise error and imaging system error can be reduced or eliminated through such simple methods.

The specific operating steps of the proposed method were as follows: (1) a grid was fabricated on the tested object surface with a particular frequency that satisfies the measurement requirement. (2) An appropriate image acquisition device with an appropriate camera lens was selected to clearly observe grids. Steps were taken to ensure that the focus was not lost during deformation and after the specimen had been rotated. (3) The grid image was acquired before deformation and rotation, which was selected as the first reference image. (4) The specimen was fixed on the specimen table (5D adjustable platform). The direction of the two grid lines were parallel or vertical to the rotation axis of the specimen table. The tested specimen was rotated around the rotation axis by a specific angle, and the current grid figure was used as the second carrier reference image. (5) The specimen was loaded, and the grid images sequence during deformation was acquired in real time. (6) The obtained grid images were decoupled through fast Fourier transform and inverse transform to acquire two unidirectional grid images that corresponded to the observation angles of the view and to calculate the 'virtual' displacement field $U^{\prime}\left(x_{i}, y_{j}\right)$ and the displacement field $V^{\prime}\left(x_{i}\right.$, $y_{j}$ ). Thus, the 3D deformation distribution at different loading times could be obtained.

It should be ensured that whether the deformation is centrosymmetric or not before the measurement, which can be judged through two conditions: (1) the shape of loading region of the tested specimen is centrosymmetric and (2) the applied loading is uniformly distributed. A microscale static feasibility test and a high-speed impact experiment that simulates an underwater explosion are conducted to verify the efficiency and accuracy of the developed method. 


\section{Feasibility Experiments}

\section{Static 3D Deformation Measurement in Microscale}

A sub-microscale 3D static deformation test was completed in a SEM, in which a 1200 line/mm holographic orthogonal grating was used as a specimen grid. If such a holographic orthogonal grating was placed in a humid environment for a long period, then bulge damage would occur because of the oxidation of photoresist, which appears as a circular bulge on the grid surface with a diameter of tens of micrometers. Thus, a deformation measurement on the grid surface is necessary to evaluate the grid quality, with most bulges satisfying the requirements of centrosymmetry. The specific steps of the procedure are as follows: the unbroken holographic orthogonal grating on which bulge occurs was fixed on the specimen table, and the central region of a tested bulge was placed near the center of whole field of view in SEM. The magnification times and working distance were adjusted to obtain a clear SEM image. The specimen table was rotated at an angle $\varphi$, and the SEM images were then acquired. The experimental view is shown in Fig. 5(a). The SEM image captured after the specimen was rotated by $10^{\circ}$ and was decoupled; two unidirectional grid lines were obtained, as shown in Fig. 5(b) and (c). The two grid lines were used to calculate the coupled displacement field $U^{\prime}\left(x_{i}, y_{j}\right)$ and displacement field $U\left(x_{i}, y_{j}\right)$, and the displacement field $U\left(x_{i}, y_{j}\right)$ was obtained by rotating the displacement field $V^{\prime}\left(x_{i}, y_{j}\right)$ around the symcenter by $90^{\circ}$, as shown in Fig. 5(d) and (e). The 3D morphology of the bulge can be reconstructed and calculated by using equation (7), as shown in Fig. 5(f). Compared with the 3D SMM (3D SEM moiré) results [12], the error of the highest point in the calculation result was approximately $2 \%$. The following computational formula was used to measure sensitivity:

$$
\begin{aligned}
h\left(x_{i}, y_{j}\right) & =\frac{U^{\prime}\left(x_{i}, y_{j}\right)-U\left(x_{i}, y_{j}\right) \cos \varphi}{\sin \varphi} \\
& =\frac{\left[N_{\mathrm{U}^{\prime}}\left(x_{i}, y_{j}\right)-N_{U}\left(x_{i}, y_{j}\right) \cos \varphi\right] p}{\sin \varphi}
\end{aligned}
$$

where $p=830 \mathrm{~nm}$ and $N_{U^{\prime}}\left(x_{i}, y_{j}\right)=N_{U}\left(x_{i}, y_{j}\right)=1$, the rotation angle of the specimen was $10^{\circ}$, and the measurement sensitivity of the out-of-plane height was $72.62 \mathrm{~nm}$ (the grid pitch is $830 \mathrm{~nm}$ ). The aforementioned sensitivity meets the experimental requirements. The experimental results and analysis has verified the accuracy of this technique.

\section{D Dynamic Deformation Measurement under a Shock Load}

The process of high-speed dynamic deformation of a sample panel was investigated in this study, which could resemble impulsive loading to simulate underwater explosions. The experimental equipment and optical paths are presented in Fig. 6. The scaled Fluid-structure Interaction (FSI) experimental setup (as in Fig. 6(a)) is similar to that used by Espinosa et al. [19] which uses a laboratory setting for underwater explosive loading conditions. In the FSI setup, a water chamber made of a steel tube was incorporated into a gas gun apparatus [37]. The specimen panel and a $22 \mathrm{~mm}$ thick piston

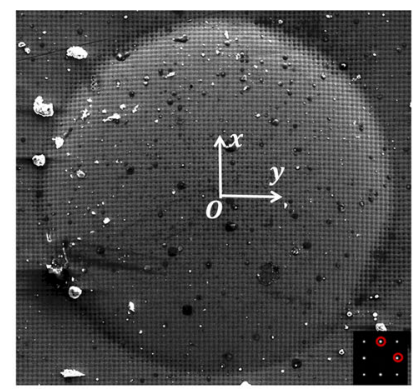

(a)

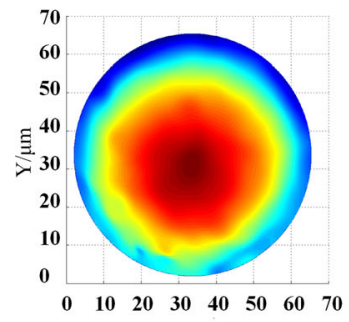

(d)

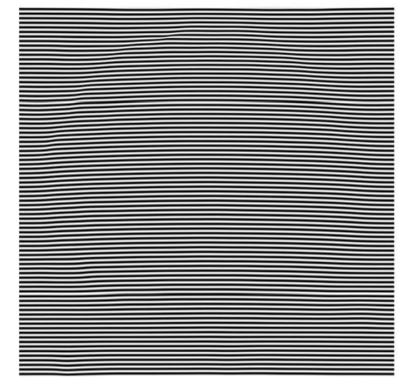

(b)

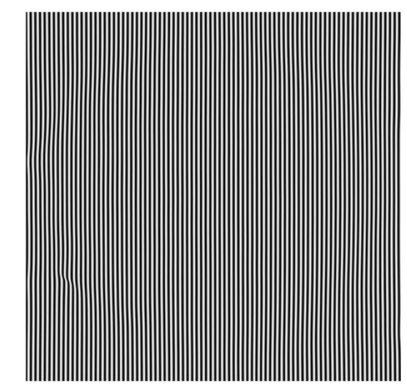

(c)

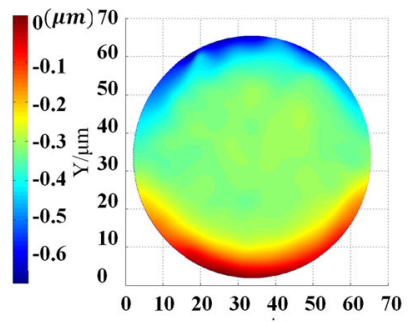

(e)

Fig. 5 Experimental results showing: (a) a SEM image of a holographic orthogonal grating captured after the specimen is rotated by $10^{\circ}$ along the oy axis; (b) and (c), the unidirectional grid images after decoupling; (d)

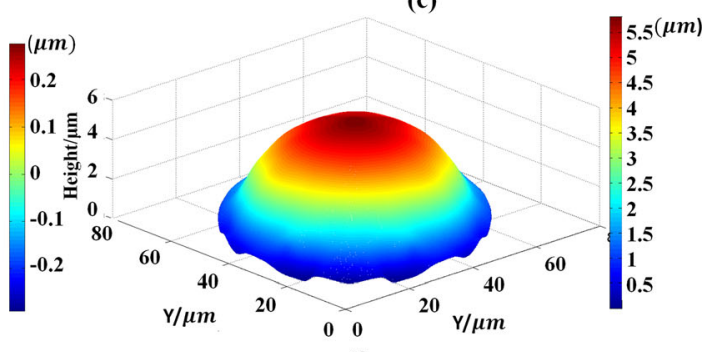

(f)

the coupled displacement field $U^{\prime}\left(x_{i}, y_{j}\right)$; (e) the displacement field $U\left(x_{i}, y_{j}\right)$; and (f) the reconstructed $3 \mathrm{D}$ topography 


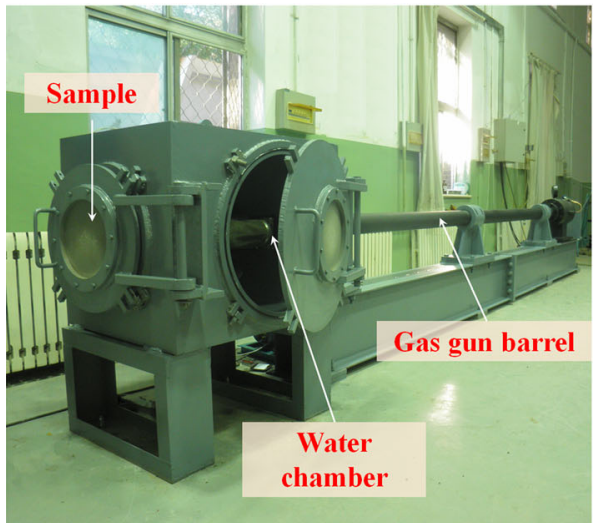

(a)

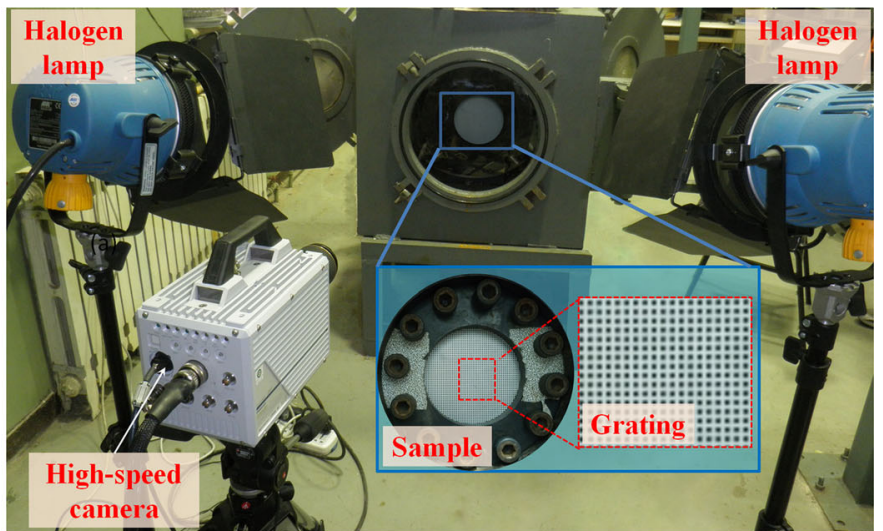

(b)

Fig. 6 Photos showing: (a) the experimental equipment used for simulating underwater explosions; and (b) the optical path of the experiment

were installed at the rear and front ends. Exponentially decaying pressure was produced by impacting the piston with a $5 \mathrm{~mm}$ thick flyer plate launched by a gas gun [37]. More details on the experimental principles and the impact process of pulse loading can be found in the literature [19, 37]. The experiment used an aluminum plate as the sample panel and a single-camera and grid to measure the dynamic 3D deformation under impulsive loading. The thickness of the aluminum plate was $3.0 \mathrm{~mm}$ and the diameter of the specimen exposed to the water blast pressure was $152.4 \mathrm{~mm}$. To obtain a clamped boundary condition, 12 ring spacers were inserted to prevent core crushing during the clamping process, as shown in Fig. 6(b).

The specific steps for the experiment were as follows: a flat lacquer was uniformly sprayed on the rough aluminum plate surface. Laser marking was used to manufacture a grid of
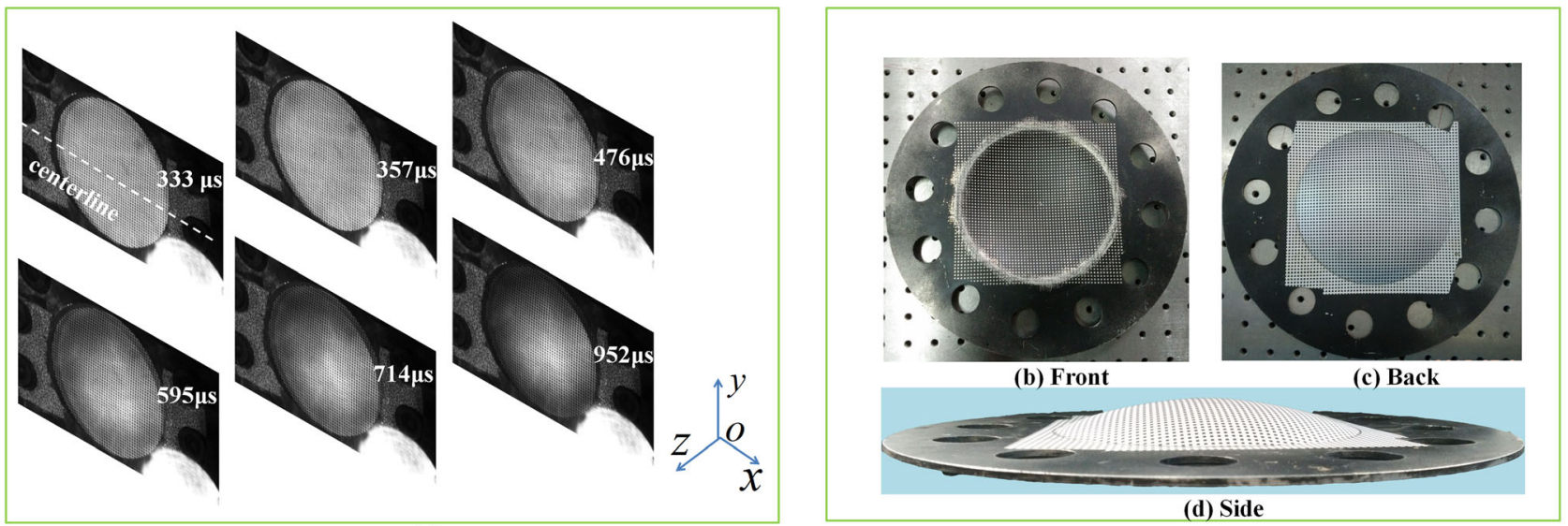

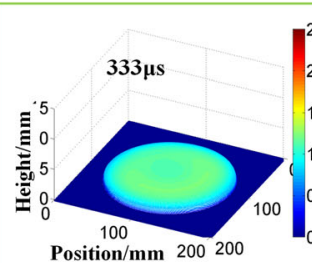

(e)

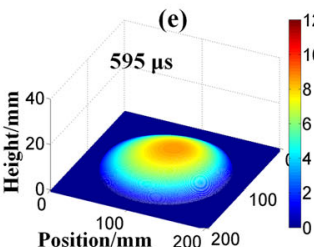

(h)
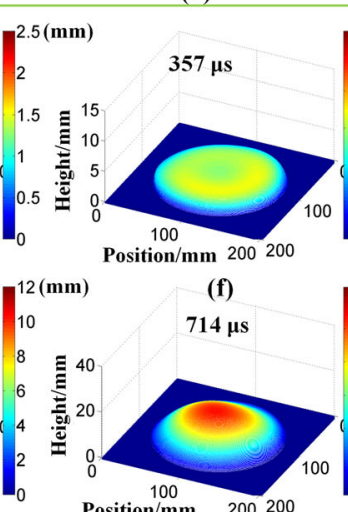

Position $/ \mathbf{m m}$
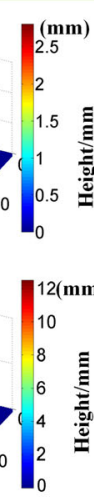

(i)

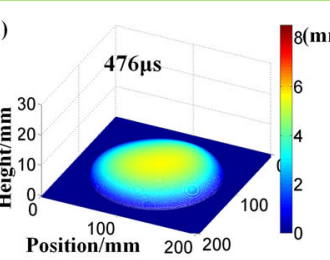

$(\mathrm{g})$

(g)

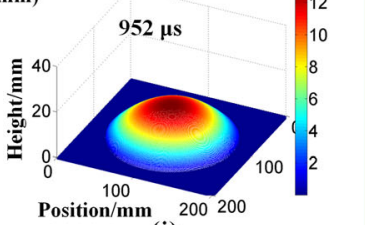

(j)

field height displacements at different time steps for the plate; and $(\mathbf{k})$ is the displacement profile along the $x$-axis

Fig. 7 Experimental results showing: (a) the deformed grid sequence during a high-speed impact experiment; (b), (c), and (d), the front, back, and side of the plate; (e), (f), (g), (h), (i), and (j), the typical full-

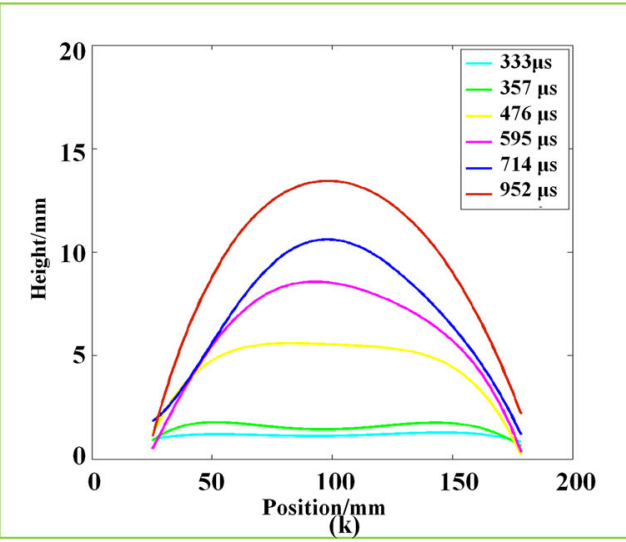


$3 \mathrm{~mm} /$ line, as shown in Fig. 6(b); this step was significant because the experimental results could be affected if the flat lacquer falls off during the process. The grid line direction of the grid was adjusted to a position parallel and vertical to the ground, and the specimen was clamped using a steel ring with 12 screws at the rear of the anvil tube. The optical axis of the camera was adjusted in the horizontal direction using a gradienter, and the high-speed camera was positioned behind the anvil tube with a viewing angle of $14^{\circ}$ to record the grid images on the back of the sheet, as shown in Fig. 6(b). The central region of tested aluminum plate was placed near the center of the whole field of view. A complete optical path was established, along with the parameter adjustment of instruments, thereby ensuring that the camera and the bullet were triggered simultaneously. The experiment was then initiated. The experimental instruments included a Photron SA-4 highspeed camera and two halogen lamps, and the parameters were as follows: a frame rate of 42,000 frames/s (an interframe time of $23.8 \mu \mathrm{s}$ ) was set with an image resolution of $512 \times 320$ pixels for $1 \mathrm{~s}$ time duration, with the impact velocity of the flyer at $90 \mathrm{~m} / \mathrm{s}$.

During impulsive loading, the sequence images of the grid on the sample panel surface were captured as shown in Fig. 7(a), which were used to calculate the profile deformation process of the sample panel. The measured out-of-plane displacements are shown in Fig. 7(e)-(j), and the cross-section variation of the profile is shown in Fig. 7(k). Figure 7(e)-(f), and $(\mathrm{k})$, show that the deformation of the sample panel during impulsive loading experienced a process from edge to center, and the shape of the sample panel at the first moments is similar to the "M"-shaped revolved body, namely, the center pits. The bulging parts were increasingly closer to the center, and the center pits began to plump up; the out-of-plane displacement reached a peak at $952 \mu$ s. This phenomenon was similar to that reported in [37], in which a pair of high-speed cameras was used.

Compared with the maximum value of the out-of-plane deformation measurement using a laser displacement sensor after deformation, the result obtained using the method developed here had an error of less than $5 \%$. The height measurement sensitivity in this experiment was $369 \mu \mathrm{m}$ (the grid pitch is $3 \mathrm{~mm}$ ). The feasibility experiments verified that the developed method could be applied not only in microscale, but also in high-speed dynamic deformation measurement with only one camera, which avoids the non-synchronization problem.

\section{Conclusions}

To meet the measurement requirements of centrosymmetric deformations, a multi-scale method for static and dynamic 3D deformation measurements based on a single-camera and grids have been proposed. The theoretical equation of this technique was deduced. This technique not only reduces the cost of the experiment, but also solves most of the problems caused by non-synchronization of time in using a pair of highspeed cameras during dynamic measurement.

On the basis of theoretical analysis and experimental feasibility verification, this study confirms the feasibility of profile measurements using a single-camera and grids. A confirmatory experiment of holographic grating bulge deformation proved the effectiveness of this method in a micro 3D static deformation measurement, in which surface topography was measured. Compared with the morphological results measured by 3D SMM, the results obtained through the proposed method have a $2 \%$ error. Sample panel 3D deformation measurement in a simulated underwater explosion experiment confirmed the effectiveness of this approach in a high-speed impact experiment compared with experimental results in literature $[19,37]$. The centerline deformation processes of the sample panel were identical. The error of the measured deformation was less than $5 \%$. Two experiments verified the features of this method; these features are minimal equipment, simplicity and convenience, and can be used to measurement centrosymmetric 3D deformation of multi-scale from static to dynamic, among others. The use of only one camera avoids the non-synchronization problem that occurs when a pair of high-speed cameras are used during common high-speed 3D measurements. Thus, this method is practical for multi-scale static and dynamic centrosymmetric 3D deformation measurement.

Acknowledgments The authors are grateful to the financial support from the National Natural Science Foundation of China (11232008, 11572041 and 11372037),the Program for New Century Excellent Talents in University (NCET-12-0036) and the Opening fund of State Key Laboratory of Nonlinear Mechanics.

\section{References}

1. Roncaglia A, Ferri M (2011) Thermoelectric materials in MEMS and NEMS: a review. Sci Adv Mater 3(3):401-419

2. Liu WK, Karpov EG, Park HS (2006) Nano mechanics and materials: theory, multiscale methods and applications. John Wiley \& Sons, Hoboken

3. Bhushan B (2005) Nanotribology and nanomechanics. Wear 259(7):1507-1531

4. Shi Z, Feng X, Huang Y, Xiao J, Hwang KC (2010) The equivalent axisymmetric model for Berkovich indenters in power-law hardening materials. Int J Plast 26(1):141-148

5. Su D, Li X (2014) Investigation of near-surface mechanical properties of materials using atomic force microscopy. Exp Mech 54(1):11-24

6. Chasiotis I, Knauss WG (2002) A new microtensile tester for the study of MEMS materials with the aid of atomic force microscopy. Exp Mech 42(1):51-57

7. Arai Y, Yokozeki S (2006) Micro-three dimensional shape measurement method based on shadow Moiré using scanning electron microscopy. J Mod Opt 53(18):2641-2655 
8. Arai Y, Kanameishi S, Yokozeki S (2010) Three-dimensional microstructure measurement by high-resolution fringe analysis for shadow moiré image by SEM. Proc. SPIE 7790, Interferometry XV: Techniques and Analysis: 77900E

9. Arai Y, Ando M, Kanameishi S, Yokozeki S (2011) Micro 3D measurement method using SEM. Mapan 26(1):69-78

10. Sutton MA, Li N, Garcia D, Cornille N, Orteu JJ, McNeill SR, Li XD (2006) Metrology in a scanning electron microscope: theoretical developments and experimental validation. Meas Sci Technol 17(10):2613-2622

11. Zhu T, Sutton MA, Li N, Orteu JJ, Cornille N, Li X, Reynolds AP (2011) Quantitative stereovision in a scanning electron microscope. Exp Mech 51(1):97-109

12. Li CW, Liu ZW, Xie HM, Wu D (2013) Novel 3D SEM Moiré method for micro height measurement. Opt Express 21(13): 15734-15746

13. Li CW, Liu ZW, Xie HM (2013) A measurement method for micro 3D shape based on grids-processing and stereovision technology. Meas Sci Technol 24(4):045401

14. Barnard ST, Fischler MA (1982) Computational stereo. ACM Comput Surv (CSUR) 14(4):553-572

15. Beberniss T, Spottswood M, Eason T (2011) High-speed digital image correlation measurements of random nonlinear dynamic response. Exp Appl Mech 6:171-186

16. Ha NS, Vang HM, Goo NS (2015) Modal analysis using digital image correlation technique: an application to artificial wing mimicking Beetle's hind wing. Exp Mech 55(5):989-998

17. Allaeys F, Luyckx G, Sarrazin C, Van Paepegem W, Jovanov L, Philips W (2014) A 3D shape measurement technique that makes use of a printed line pattern. Exp Mech 54(6):999-1009

18. Hÿtch MJ, Plamann T (2001) Imaging conditions for reliable measurement of displacement and strain in high-resolution electron microscopy. Ultramicroscopy 87(4):199-212

19. Espinosa HD, Lee S, Moldovan N (2006) A novel fluid structure interaction experiment to investigate deformation of structural elements subjected to impulsive loading. Exp Mech 46(6):805-824

20. Berke RB, Sebastian CM, Chona R, Patterson EA, Lambros J (2016) High Temperature Vibratory Response of Hastelloy-X: Stereo-DIC Measurements and Image Decomposition Analysis. Exp Mech 56(2):231-243

21. Lee HS, Yoon JH, Park JS, Yi YM (2005) A study on failure characteristic of spherical pressure vessel. J Mater Process Technol 164:882-888

22. You LH, Zhang JJ, You XY (2005) Elastic analysis of internally pressurized thick-walled spherical pressure vessels of functionally graded materials. Int J Press Vessel Pip 82(5):347-354
23. Spranghers K, Vasilakos I, Lecompte D, Sol H, Vantomme J (2012) Full-field deformation measurements of aluminum plates under free air blast loading. Exp Mech 52(9):1371-1384

24. Veldman RL, Ari-Gur J, Clum C, DeYoung A, Folkert J (2006) Effects of pre-pressurization on blast response of clamped aluminum plates. Int J Impact Eng 32(10):1678-1695

25. Neuberger A, Peles S, Rittel D (2007) Scaling the response of circular plates subjected to large and close-range spherical explosions. Part II: buried charges. Int J Impact Eng 34(5):874-882

26. Chen X, Vlassak JJ (2001) Numerical study on the measurement of thin film mechanical properties by means of nanoindentation. J Mater Res 16(10):2974-2982

27. Vlassak JJ, Nix WD (1992) A new bulge test technique for the determination of young's modulus and poisson's ratio of thin films. J Mater Res 7(12):3242-3249

28. Cao YY, Wang C, Ma QW, Ma SP (2015) Experimental investigation of the startup time difference between high-speed cameras. Chin Opt Lett 13(7):40-44

29. Hÿtch MJ, Gandais M (1995) Quantitative criteria for the detection and characterization of nanocrystals from high-resolution electron microscopy images. Philos Mag A 72(3):619-634

30. Hÿtch MJ, Snoeck E, Kilaas R (1998) Quantitative measurement of displacement and strain fields from HREM micrographs. Ultramicroscopy 74(3):131-146

31. Liu ZW, Xie HM, Gu CZ, Meng YG (2008) The digital geometric phase technique applied to the deformation evaluation of MEMS devices. J Micromech Microeng 19(1):015012

32. Liu ZW, Lou XH, Gao JX (2010) Deformation analysis of MEMS structures by modified digital moiré methods. Opt Lasers Eng 48(11):1067-1075

33. BIPM, IEC, IFCC, ILAC, IUPAC, IUPAP, ISO, OIML (2008) Evaluation of measurement data - guide for the expression of uncertainty in measurement. JCGM 100:2008

34. Yoneyama S, Kitagawa A, Kitamura K, Kikuta H (2006) In-plane displacement measurement using digital image correlation with lens distortion correction. JSME Int J Ser A: Solid Mech Mater Eng 49(3):458-467

35. Schreier HW, Garcia D, Sutton MA (2004) Advances in light microscope stereo vision. Exp Mech 44(3):278-288

36. Shi WX, Huang XF, Liu ZW (2014) Transmission-lattice based geometric phase analysis for evaluating the dynamic deformation of a liquid surface. Opt Express 22(9):10559-10569

37. Xiang DL, Rong JL, He X (2015) Experimental investigation of dynamic response and deformation of aluminum honeycomb sandwich panels subjected to underwater impulsive loads. Shock Vib 2015:1-13 